\title{
Intrapulmonary agenesis of venous system and bronchopulmonary arterial anastomosis
}

\author{
Mohinder K Thapar, Eduardo Riff, Zohair Halees
}

\begin{abstract}
Agenesis of the intrapulmonary and extrapulmonary veins of the right lung was found by angiography and histological examination in a three year old boy. Blood supplied by the intersegmental arteries drained via the pulmonary arteries of the right lung into main and left pulmonary arteries. This caused a shunt between the aorta and pulmonary artery. Agenesis of the pulmonary venous system as a cause of left to right shunt has not been reported before.
\end{abstract}

Several forms of pulmonary venous abnormalities, such as pulmonary vein stenosis or atresia $^{12}$ and total or partially abnormal pulmonary venous connections ${ }^{3}$ to various sites, have been described. However, the complete congenital absence or agenesis of intrapulmonary and extrapulmonary veins of one lung has not been reported before. There are 17 reports of a fistulous communication between the internal mammary or intercostal artery and the pulmonary artery ${ }^{4-6}$; but all of these cases had normal pulmonary venous return. We report a child with complete agenesis of the intrapulmonary and extrapulmonary veins of the right lung.

\section{Case report}

CLINICAL FEATURES

A three year old boy presented with a history of recurrent lung infections since birth. There were no other cardiovascular or respiratory symptoms. Growth and development were normal. The perinatal and family histories were unhelpful.

He had normal vital signs and no cyanosis. His height and weight were in the tenth percentile. Peripheral pulses were normal with a

Results of cardiac catheterisation

\begin{tabular}{lllc}
\hline Site & $\begin{array}{l}\text { Saturations } \\
(\%)\end{array}$ & $\begin{array}{l}\text { Pressures } \\
(\mathbf{m m} \mathrm{Hg})\end{array}$ & $\begin{array}{l}\text { Mean } \\
(\mathbf{m m} \mathrm{Hg})\end{array}$ \\
\hline Superior vena cava & 73 & & \\
Right atrium & 66 & $2-7, \mathrm{v}-5$ & 4 \\
Right ventricle & 65 & $27 / 0-6$ & 18 \\
Main pulmonary artery & 86 & $23 / 12$ & 18 \\
Left pulmonary artery & 80 & $22 / 12$ & 18 \\
Right pulmonary artery & 96 & $24 / 11$ & 59 \\
Left ventricle & 98 & $74 / 0-8$ & $51 / 4$ \\
Aorta & 99 & $73 / 41$ &
\end{tabular}

Assumed oxygen consumption $=140 \mathrm{ml} / \mathrm{min}$; cardiac index $=3.41 / \mathrm{min} / \mathrm{m}^{2} ;$ pulmonary index Assumed ox

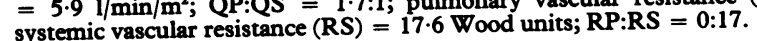

blood pressure of $100 / 50 \mathrm{~mm} \mathrm{Hg}$ in the right arm. Cardiac findings included an apical impulse in the fourth left intercostal space inside the midclavicular line. No heave or thrill were present. On auscultation first and second heart sounds were normal. No systolic or diastolic heart murmurs were present. Examination of the chest showed decreased breath sounds over the right hemithorax. There was no hepatosplenomegaly. The rest of the physical examination was unremarkable.

\section{INVESTIGATIONS}

A complete blood count with differential count, sedimentation rate, urine analysis, hepatic and renal profiles, serum $\gamma$ globulins, tuberculin skin test, and sweat chloride test were negative. Multiple blood and sputum cultures were sterile. The arterial blood gas profile while he was breathing room air showed $\mathrm{pH} 7 \cdot 38, \mathrm{PaCO}_{2} 37$, and $\mathrm{PaO}_{2} 87$ $\mathrm{mm} \mathrm{Hg}$.

\section{Chest $x$ ray}

The trachea and mediastinum were shifted to the right side. The right hemidiaphragm was raised. There were diffuse infiltrates and pleural thickening in the right lung. Bronchography showed absence of the posterior segment (RB2) of the right upper lobe. The right middle lobe was normal. Except for the basilar segment (RB9), the other segmental bronchi of the right lower lobe were clustered together and were much smaller in diameter and length than normal. A ventilation and perfusion lung scan showed decreased ventilation and no perfusion of the right lung. The left side was normal.

\section{Electrocardiogram}

There was left axis deviation but the electrocardiogram was otherwise normal for age. Cross sectional echocardiography with Doppler studies showed a reverse flow pattern in the right pulmonary artery and a normal cardiac anatomy.

\section{Cardiac catheterisation}

The pressures in the right and left sides of the heart were normal (table). There was a stepup in oxygen saturation in the main and left pulmonary arteries. The oxygen saturation in the right pulmonary artery was $99 \%$. The calculated QP:QS was 1·7:1 (assumed oxygen consumption $140 \mathrm{ml} / \mathrm{min}$ ). Systemic and pulmonary resistances were normal. 
Figure 1 Venous phase of arterial angiograms.

(A) Dye was injected into the systemic artery

supplying the right lung.

This showed the capillary phase and retrograde filling of the pulmonary arterial tree. Dye flowed

retrogradely from the right to the left pulmonary artery. (B) Dye was injected into the main pulmonary artery. During laevophase the left pulmonary veins were seen entering the left atrium. No pulmonary veins were seen on the right side.
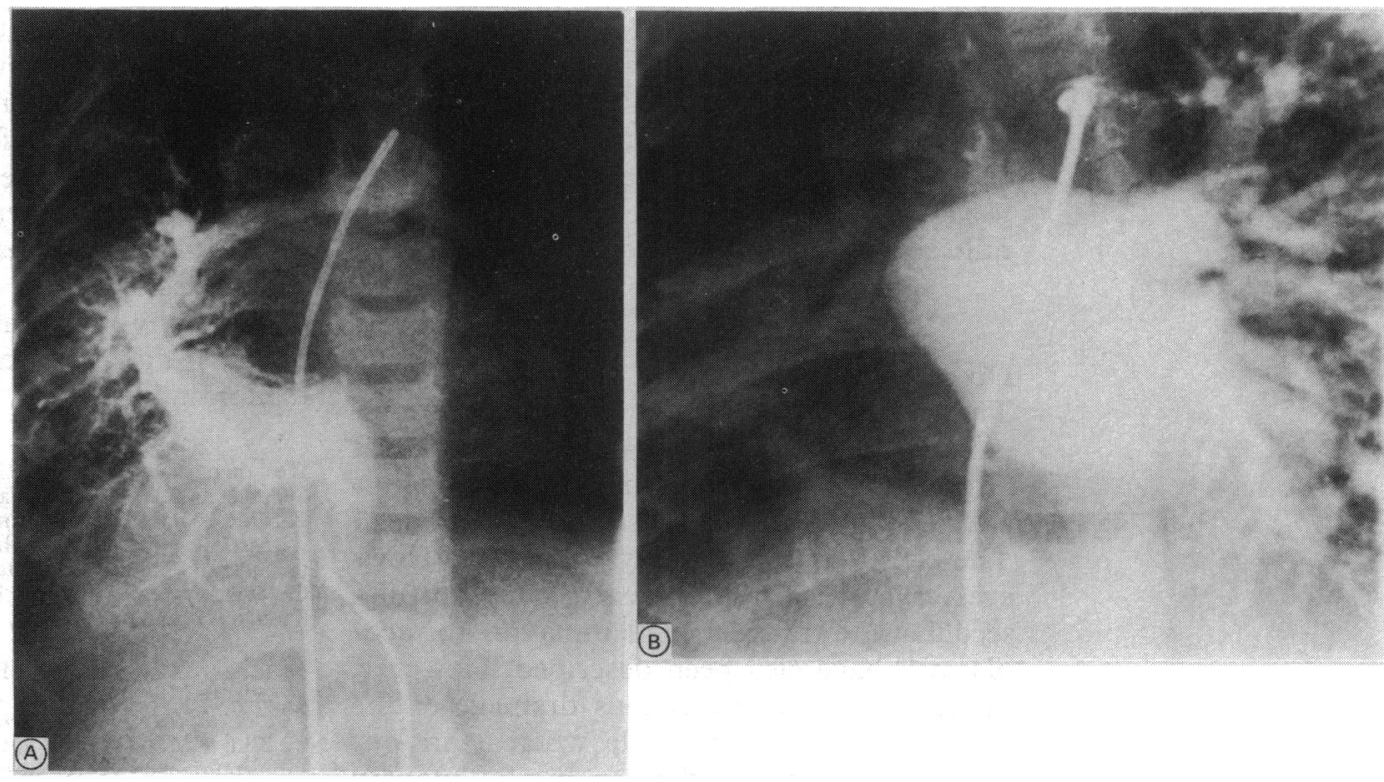

Cineangiography

The main pulmonary artery angiogram showed that there was a continuous flow of unopacified blood from the peripheral to the central right pulmonary artery and then into the main and left pulmonary arteries. Dye selectively injected into the distal portion of the right pulmonary artery was washed back via the central right pulmonary artery into the main and left pulmonary arteries. There was no forward flow into the capillaries and veins of the right lung. The left lung was normal. An aortic angiogram showed that systemic arteries, two from the thoracic aorta and one from the abdominal aorta, supplied the right lung. The selective angiograms from these arteries showed the flow into the capillary bed of the right lung. During the laevophase of these angiograms (fig 1) dye collected into the pulmonary arterial tree and then left the lung via the right pulmonary artery into the main and left pulmonary arteries. Neither right pulmonary nor systemic arterial injections showed a venous system from the right lung.

\section{Surgery}

Right pneumonectomy was performed. Three systemic vessels were identified: two arising from the thoracic aorta entered the hilar region and one from the abdominal aorta entered through the diaphragm into the base of the lung. No pulmonary veins, fibrous strands, or cord connected the right lung to the left atrium.

\section{HISTOLOGY}

We examined serial sections made of tissue at various levels of the formalin fixed, uninflated right lung. Systemic elastic arteries entered the right lung, two in the hilar region and one on the diaphragmatic surface of the lung. These vessels ended in a plexus of capillaries close to the airways and acini. The pulmonary arteries also anastomosed with this plexus. The muscular pulmonary arteries, predominantly in the lower lobe, showed changesHeath and Edwards classification types I and II. An occasional artery showed plexiform lesions and venous-like dilatations. There was

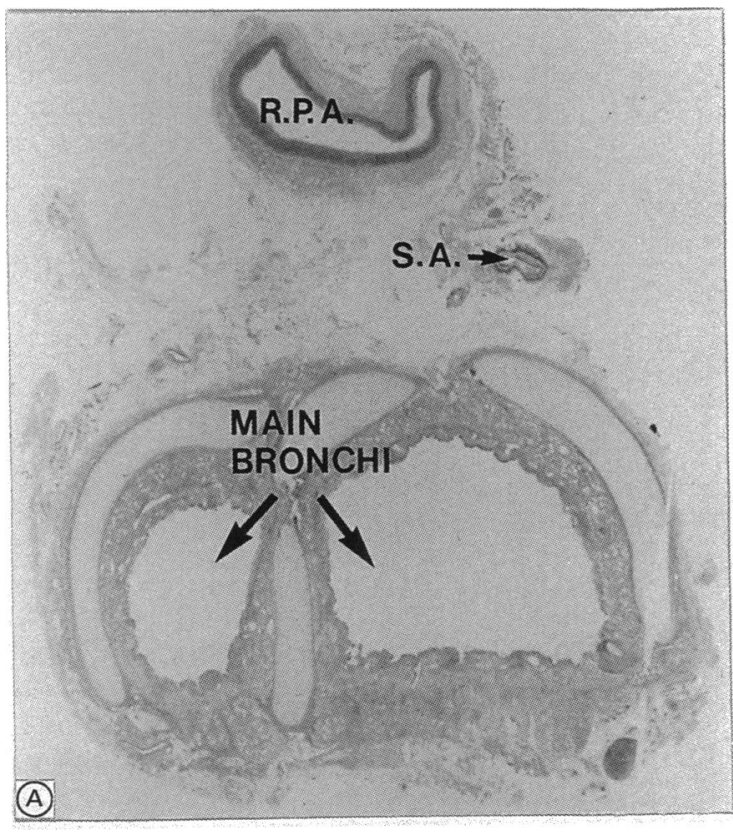

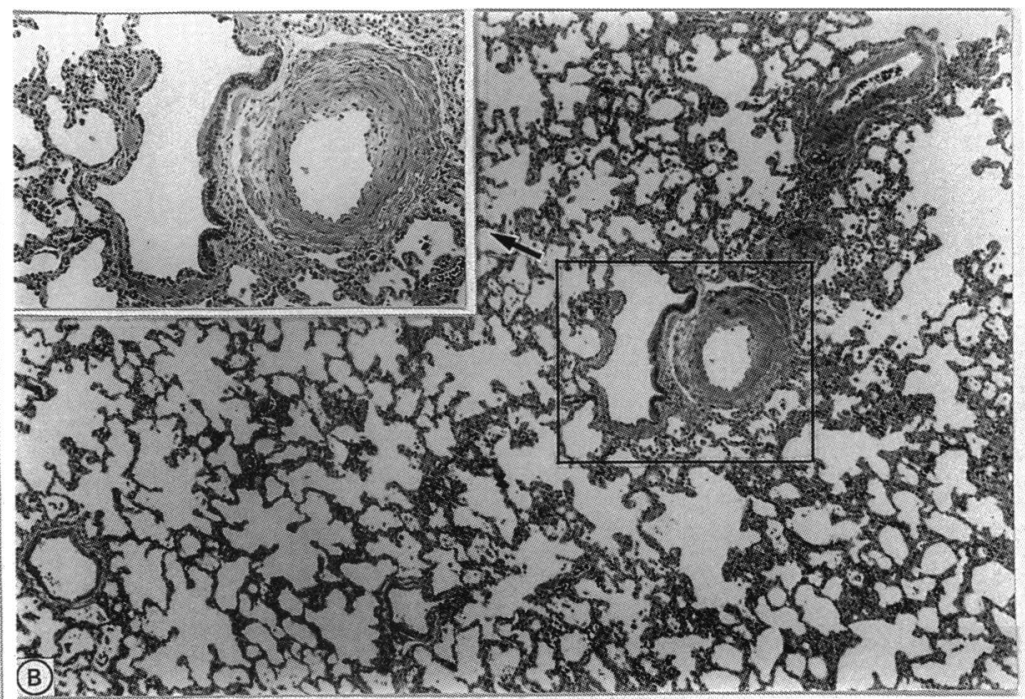

Figure $2 A$ histological section of the right lung: $(A)$ from the hilar region of the Figure $2 A$ histological section of the right lung: $(A)$ from the hilar region of the lung showing that the bronchus and the artery had no accompanying bronchial or pulmonary veins. $R P A$, right pulmonary artery; $S A$, small artery. 
no right pulmonary vein. We found no pulmonary or bronchial veins accompanying the pulmonary arteries and airways (fig 2). The airways and acini seemed to be normal except for occasional foci of early emphysematous lesions. We also found irregularly distributed, mild septal, interstitial, and pleural fibrosis.

\section{Discussion}

There are 17 case reports of systemic to pulmonary artery anastomosis; in most a right internal mammary to pulmonary artery fistula affected either a lobe or a segment of a lobe..$^{4-6}$ These fistulas were mostly congenital. However, systemic to pulmonary artery fistulas secondary to trauma, ${ }^{6-8}$ inflammation, ${ }^{9}$ or neoplasms ${ }^{10}$ have also been described. However, in all these patients, venous drainage of the affected lobe was normal; whereas in our patient we found neither an intrapulmonary nor extrapulmonary venous system of the right lung by angiography or histology.

The venous system of the lungs develops from two different sources. ${ }^{11}$ Initially, the splanchnic plexus arising from the foregut provides the venous drainage into the systemic veins for the developing pulmonary capillaries. Later, with the development of the common pulmonary vein and absorption of the systemic venous connection, pulmonary venous drainage is established into the left atrium. We speculate that in early stages of development the failure of the splanchnic system to connect with the developing pulmonary capillaries of the right lung mesenchyma resulted in the persistence of the anastomosis between the intersegmental and pulmonary arteries. Postnatally this anastomosis led to a systemic to pulmonary artery shunt. The absence of pulmonary veins in the lung has not been reported before.

Non-invasive investigations in this child did not show the true nature of the abnormality, whereas cineangiography and cardiac catheterisation did. We recommend cineangiography and cardiac catheterisation in patients with suspected pulmonary vascular malformations.

We thank Dr F Rank, Department of Pathology and Sengodagounder Balasundaram, FRCS, Department of Cardiovascular Surgery, King Faisal Specialist Hospital and Research Centre, for their help; Dr M Rabinovich, Cardiovascular Pathology, Hospital for Sick Children, Toronto Canada for reviewing the histological sections; and Dr F Kutayli for guidance.

1 Lucas RV, Woolfrey BF, Anderson RC, Lester RG, Edwards JE. Atresia of the common pulmonary vein. Edwards JE. Atresia of

2 Bini RM, Cleveland DC, Celeallos R, et al. Congenital pulmonary vein stenosis. Am J Cardiol 1984;54:369-75.

3 Kingston HM, Patel RG, Watson GH. Unilateral absence or extreme hypoplasia of pulmonary veins. Br Heart 1983;49:148-53.

4 Hirsch M, Maroko I, Gueron M, Goleman L. Systemicpulmonary arteriovenous fistula of traumatic origin: a case report. Cardiovasc Intervent Radiol 1983;6:160-3.

5 Izumi S, Okamoto M, Beppu S, et al. Intercostal artery to pulmonary artery fistula. Am J Cardiol 1984;54:688-9.

6 Fein KB, Godwin JD, Moore AV, Moran JF, Young WG Systemic artery-to-pulmonary vascular shunt: a comSystemic artery-to-pulmonary vascular shunt: a com-
plication of closed-tube thoracostomy. Am J Radiol plication of closed

7 Cox PA, Keshishian JM, Blades BB. Traumatic arteriovenous fistula of the chest wall and lung secondary to insertion ous fistula of the chest wall and lung secondary to insertion
of an intercostal catheter. $J$ Thorac Cardiovasc Surg of an intercosta

8 Saito T, Matsuda M, Yamaguchi T, Matsuzaki T, Nagakawa S, Akatsuka N. A case of traumatic systemic-pulmonary arteriovenous fistula. Jpn Heart J 1975;16:196-203.

9 Ishikawa T. Systemic artery-pulmonary artery communication in Takayasu's arteritis. Am J Roentgenol 1977 128:389-93.

10 Dunn RP, Wexler L. Systemic-to-pulmonary fistula in intrapulmonary Hodgkin's disease. Chest 1972;66:590-4.

11 Spencer H. Pathology of the lung. 4th ed. New York: Pergamon Press, 1985:13-16. 